\title{
Hemangiomas múltiples gigantes: respuesta al tratamiento con corticoides
}

\author{
C. Madrigal Díez ${ }^{\mathrm{a}}$, A. Bidonga ${ }^{\mathrm{b}}$, V. Madrigal Díez ${ }^{\mathrm{c}}$ \\ aPediatra. CS Cazoña. Santander. España. \\ bClínica Pediátrica San José en Bor. Bissau. Guinea Bissau. \\ cServicio de Pediatría. Hospital Universitario Marqués de Valdecilla. Santander. España.
}

\begin{abstract}
Resumen
La historia natural de los hemangiomas infantiles suele presentar una primera fase de crecimiento rápido seguida de otra con involución lenta y espontánea. Por este motivo el tratamiento no está indicado en la mayoría de los casos. Sin embargo, el tratamiento está justificado cuando estas lesiones interfieren con las funciones vitales del niño o producen daños estéticos importantes. En la actualidad existen diferentes opciones terapéuticas, pero los corticoides son el tratamiento más utilizado en la práctica clínica, con resultados bastante efectivos. Presentamos el caso de una niña de 7 meses que sufría una grave desfiguración por la presencia de hemangiomas múltiples gigantes y que fue tratada con éxito con corticoides sistémicos.
\end{abstract}

Palabras clave: Corticoides. Hemangioma. Tratamiento.

Multiple giant hemangiomas: response to treatment with corticosteroids

Abstract

The natural history of hemangiomas is a slow involution after a period of rapid growth.

Despite the benign course of most cutaneous hemangiomas, a significant number will cause functional compromise or permanent disfigurement. In these instances, treatment is clearly indicated.

Although there are many widely used therapeutic options available, systemic corticosteroids are generally accepted to be first line-therapy for those hemangiomas that cause complications or significant disfigurement. We present the case of a seven-month-girl with problematic cutaneous hemangiomas that caused her a serious disfigurement. She received systemic corticosteroid treatment with good response.

Key words: Hemangioma. Steroids. Therapy.

Carmen Madrigal Díez: c.madrigaldiez@yahoo.es

Los autores declaran no presentar conflictos de intereses en relación con la preparación y publicación de este artículo. 


\section{Introducción}

Los hemangiomas son los tumores benignos más frecuentes durante la infancia y son los tumores más comunes en cabeza y cuello en el niño. La incidencia global de los hemangiomas en la edad pediátrica es de aproximadamente un $10 \%$ y son 3 veces más frecuentes en niñas que en niños. Habitualmente se presentan como una lesión única, pero hasta en un $15-20 \%$ de los casos son multifocales. La cara, el cuero cabelludo, la espalda y la zona anterior del tórax son las zonas más afectadas.

Los hemangiomas suelen estar ausentes en el recién nacido y aparecer en las primeras semanas o meses de vida a partir de una marca pálida o rosada. Crecen durante el primer año adquiriendo un color rojo vivo y un mayor volumen, $y$ suelen alcanzar su mayor tamaño entre los 6 y los 12 meses de vida, pudiendo llegar a ser muy deformantes.

A partir del año entran en una fase de involución lenta, de manera que aproximadamente el $50 \%$ se ha resuelto a la edad de 5 años, el $70 \%$ a los 7 años, y el resto de lesiones se resuelve entre los 10 y los 12 años. Generalmente suelen persistir cicatrices o piel redundante en el lugar del hemangioma.

La mayor parte de los hemangiomas infantiles se diagnostican por la clínica, a través de la historia del paciente y su examen físico. Solamente es necesario realizar estudios de imagen en un reducido número de casos, generalmente ecografía o resonancia magnética, siendo excepcional la necesidad de llevar a cabo una biopsia.

Como la mayoría involucionan espontáneamente, su tratamiento debe reservarse para aquellos que, por su tamaño o localización, originen deformidades importantes y riesgo de complicaciones.

\section{Caso clínico}

Niña de 7 meses fruto de un embarazo sin incidencias y parto normal a término, residente en Guinea Bissau. A la semana de vida se advirtió la aparición de lesiones rojas y prominentes, de crecimiento rápido y confluente, que no presentaba al nacimiento. Una de las lesiones afectaba al labio inferior, mentón y cuello; otra se localizaba en la región preauricular derecha; y la tercera estaba localizada en el glúteo derecho. Su crecimiento se estabilizó en 4 meses, apareciendo en ese momento algunas úlceras. Durante el seguimiento clínico no se apreció en ningún momento signos de dificultad o compromiso respiratorio. En la exploración física a los 7 meses de edad se apreciaban 3 angiomas de gran tamaño, de superficie mamelonada y 
color rojo vivo, de consistencia firme a la palpación y con algunas zonas de ulceración y fibrosis incipiente, con bordes bien definidos. Uno abarcaba la pared inferior de la cara y anterior del cuello, originando deformación grotesca, el segundo toda la región glútea derecha y genitales, y el menor el lóbulo de la oreja y la zona preauricular derecha (figura 1). Debido a la deformidad producida por los hemangiomas se instauró tratamiento con prednisona oral, $2 \mathrm{mg} /$ $\mathrm{kg}$ /día, observándose una regresión significativa de las lesiones en 4 semanas, retirando la medicación de forma progresiva en otras 4 semanas sin apari- ción de un efecto rebote. Se obtuvo una respuesta rápida y favorable, con regresión total de las lesiones y notable mejoría estética, aunque incompleta al quedar zonas de piel cicatricial y redundante (figura 2).

\section{Discusión}

Los hemangiomas infantiles son un motivo habitual de consulta en el centro de salud por su alta incidencia en los lactantes y por la ansiedad que en algunos casos provoca a los padres que su hijo presente este tipo de malformación, sobre todo si se localiza en la cara o zonas fácilmente visibles.

Figura 1. Grandes hemangiomas en el área de la barba, en la región preauricular y en toda la región glútea derecha y genitales externos.

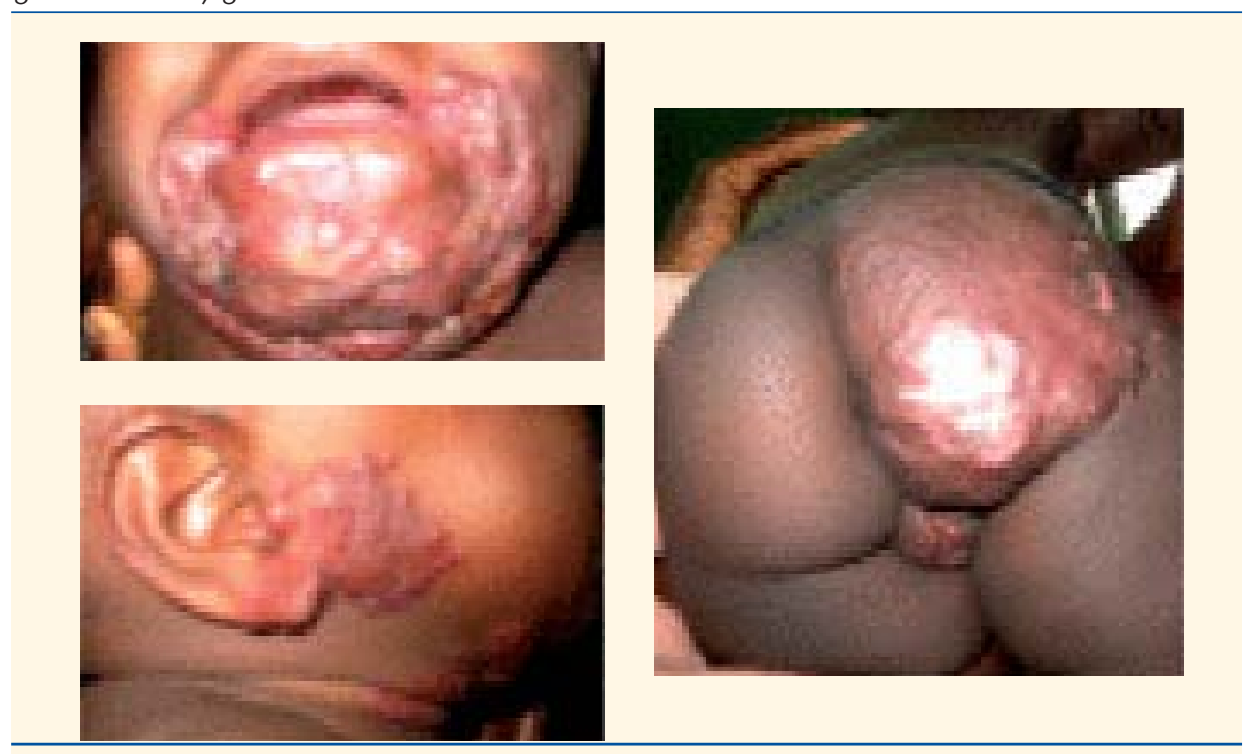


El comportamiento de un hemangioma determinado es impredecible, no guarda relación con su tamaño o localización, y muchas veces constituye un verdadero desafío para el pediatra de Atención Primaria, al que le puede ser difícil anticipar en las primeras revisiones qué hemangioma va a tener un ritmo de crecimiento inquietante o una posibilidad alta de complicaciones.

El manejo de los hemangiomas continúa siendo una cuestión controvertida, al no existir una guía terapéutica realizada a partir de estudios rigurosos basados en la evidencia, y el tratamiento debe ser individualizado teniendo en cuenta la edad, la localización anató- mica, el tamaño, la velocidad de crecimiento y otros factores ${ }^{1}$. Raramente es necesario tratarlos por comprometer funciones vitales o producir complicaciones serias y habitualmente suele ser suficiente guardar una actitud expectante mientras se observa el comportamiento de la lesión. En el caso que describimos, el tratamiento está justificado por su capacidad de producir una desfiguración grave y permanente que perturbase de forma drástica la vida de la niña. Además, el hemangioma que le afecta al labio inferior, la barbilla y la parte anterior del cuello supone un riesgo no despreciable de afectación de la vía respiratoria superior, puesto que los

Figura 2. Aspecto de las lesiones después del tratamiento con prednisona oral.

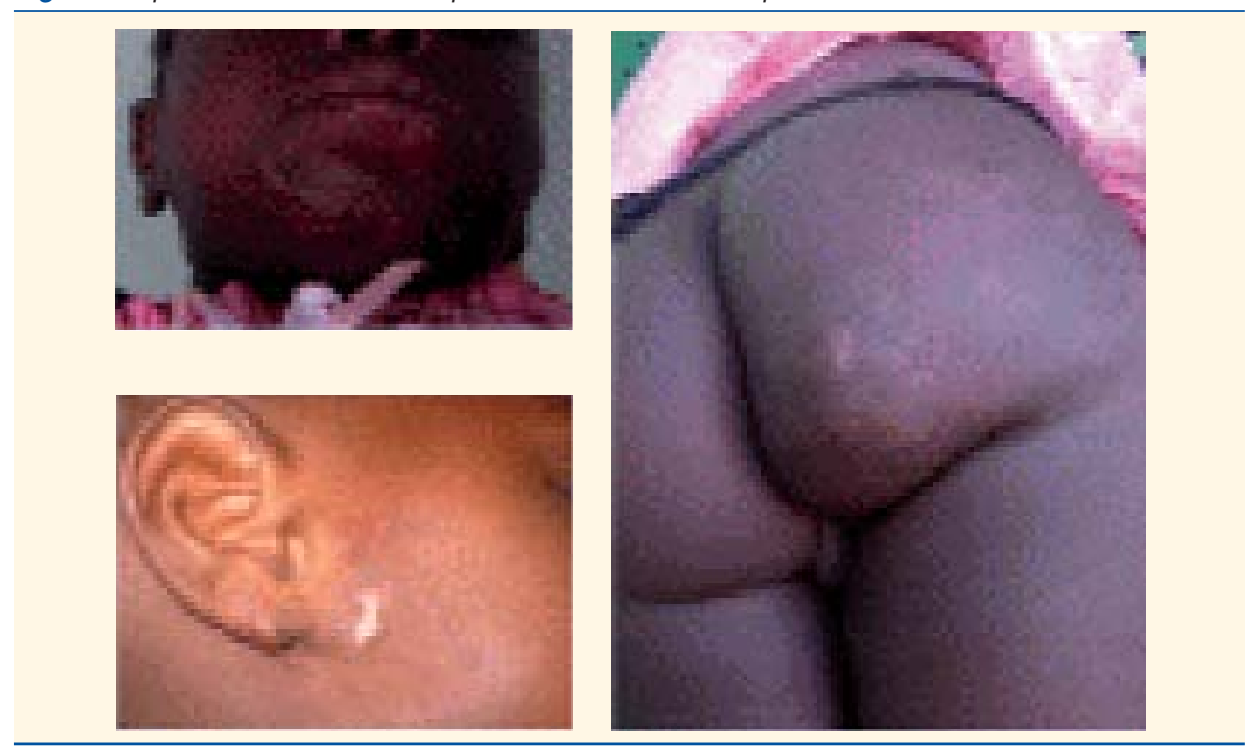


hemangiomas en el área de la barba pueden asociarse con un hemangioma de la vía aérea, la mayoría localizados en la zona subglótica². Habitualmente, los pacientes con un hemangioma en esta localización presentan síntomas antes de los 3 meses. Afortunadamente, nuestra paciente no presentó disnea, ronquera, estridor o cualquier otro síntoma que hiciera sospechar compromiso respiratorio, no siendo necesario visualizar de forma directa su vía aérea.

Los corticoides son una de las terapias más efectivas de los hemangiomas, y los corticoides sistémicos son aceptados generalmente como la primera opción terapéutica de los hemangiomas con capacidad potencial de desfiguración física, como es el caso de nuestra paciente $^{3,4}$. Otras de sus indicaciones incluyen los hemangiomas infantiles que comprometen funciones vitales, especialmente si afectan la visión o la vía respiratoria, aquellos que producen insuficiencia cardiaca congestiva y también los de gran tamaño o ulcerados.

El mecanismo por el cual los corticoides causan el cese del crecimiento de los hemangiomas es desconocido. Algunos estudios experimentales indican que la vasoconstricción es responsable de su efecto terapéutico ${ }^{5}$. Así, los corticoides no son vasoconstrictores conoci- dos, pero se cree que actúan en la resolución de los hemangiomas aumentando la sensibilidad de arteriolas y precapilares a agentes fisiológicos con acción vasoconstrictora.

Actualmente, la elección del tipo y la dosis de corticoide se basan en recomendaciones empíricas, no está establecida la dosis óptima y la duración del tratamiento, y la práctica clínica varía entre médicos. La mayoría de los autores sugieren que dosis menores de $2 \mathrm{mg} / \mathrm{kg} /$ día no son efectivas y recomiendan dosis de 2-3 mg/kg/día, que proporcionan buenos resultados con escasos y transitorios efectos secundarios a corto plazo, y sin apreciarse complicaciones significativas a largo plazo. Para ser efectivos deben administrarse cuanto antes en la fase proliferativa de crecimiento. Habitualmente se dan en dosis única matinal durante 412 semanas (tiempo que suele ser suficiente para observar una respuesta clínica), se realiza una reducción progresiva de la dosis en los siguientes meses (para evitar un efecto rebote) y se suele retirar el tratamiento antes del año de vida. Sin embargo, el tiempo de administración de los corticoides es variable y siempre hay que tener en cuenta la respuesta del paciente al tratamiento, la gravedad del hemangioma y la edad del niño cuando se inicia el tratamiento ${ }^{6}$. 
Algunos autores han descrito una mejor respuesta al tratamiento con dosis de 3-5 mg/kg/día, pero existen todavía escasos estudios que respalden esta recomendación que expone al paciente a un mayor número de posibles efectos secundarios (aunque habitualmente transitorios), sin además estar establecido un protocolo de seguimiento de los pacientes con hemangiomas infantiles que reciben corticoides en dosis altas o durante un periodo largo de tiempo.

Existen pocos estudios sobre la eficacia real de los corticoides en el tratamiento de los hemangiomas infantiles. En nuestra paciente, la eficacia del tratamiento con corticoides queda probada por la rápida regresión del los angiomas coincidiendo con la administración de la medicación y no como resultado de una involución lenta y espontánea. Un estudio sugiere que un tercio de los niños con hemangiomas tratados con corticoide oral presentan una disminución drástica de su tamaño en los días siguientes de iniciar el tratamiento, otro tercio presenta un estancamiento en el crecimiento de su hemangioma sin observarse una regresión significativa, y el otro tercio restante no tiene ningún tipo de respuesta ${ }^{7}$. Sin embargo, existen revisiones clínicas más recientes que afirman que los corticoides alcanzan una efectividad superior al $80 \%$ cuando son administrados en una dosis adecuada 8 .

Los corticoides se han utilizado en el tratamiento de distintas enfermedades crónicas inflamatorias estando bien estudiados sus efectos secundarios; sin embargo, existen pocos datos en la actualidad sobre los posibles efectos secundarios de la terapia corticoidea en el manejo de los hemangiomas infantiles. Los corticoides pueden producir irritabilidad, insomnio, lesiones gástricas, hiperglucemia, hipertensión, retraso del crecimiento, inmunosupresión y supresión de la glándula suprarrenal. Un estudio realizado en 62 pacientes tratados con corticoides sistémicos en dosis de 2-3 mg/kg/día por padecer hemangiomas problemáticos llega a la conclusión de que sus efectos secundarios a corto plazo son leves y no producen complicaciones serias a largo plazo.

En pacientes con hemangiomas agresivos en los que los corticoides sistémicos fallan o son retirados por sus efectos secundarios, se pueden probar medicaciones alternativas como la vincristina o el interferón, aunque estos pueden producir efectos secundarios neurológicos graves, sobre todo en niños menores de 12 meses $^{4}$.

En general, las lesiones no desaparecen completamente al final del trata- 
miento médico, sino que continúan involucionando durante un periodo de varios meses hasta 2 años. En los pacientes que, como en nuestro caso, presentan hemangiomas con zonas ulceradas, persisten diferentes grados de cicatrización en la piel tras la terapia ${ }^{5}$.

Uno de los aspectos más importantes para el niño afectado y sus padres es la desfiguración y las consecuencias que provoca. Un estudio realizado con hemangiomas faciales mayores de $1 \mathrm{~cm}$ de diámetro reveló que las reacciones de los padres de incredulidad, miedo y preocupación fueron frecuentes y similares en intensidad que las reacciones de los padres con un hijo con una malformación permanente ${ }^{10}$. Los padres se sentían abrumados por los comentarios de la gente y más de la mitad no estaba contento con la atención médica recibida.

El pediatra de cabecera debe estar preparado para percibir y entender el efecto que provoca el hemangioma en el niño afectado y en sus padres, procurándoles el soporte emocional adecuado e informándoles de la necesidad de un control estrecho de la evolución de la lesión, dado su carácter impredecible.

\section{Bibliografía}

1. Haggstrom AN, Frieden IJ. Hemangiomas: Past, present, and future. J Am Acad Dermatol. 2004;51:850-2.

2. Orlow SJ, Isakoff MS, Blei F. Increased risk of symptomatic hemangiomas of the airway in association with cutaneous hemangiomas in a "beard" distribution. J Pediatr. 1997;131:643-6.

3. Sundine MJ, Wirth GA. Hemangiomas: An Overview. Clin Pediatr (Phila). 2007;46:206-21.

4. Drolet BA, Esterly NB, Frieden IJ. Hemangiomas in children. N Engl J Med. 1999;341:173-81.

5. Sadan N, Wolan B. Treatment of hemangiomas of infants with high doses of prednisone. J Pediatr. 1996;128:141-6.

6. George ME, Sharma V, Jacobson J, Simon $S$, Nopper AJ. Adverse effects of systemic gluco- corticosteroid therapy in infants with hemangiomas. Arch Dermatol. 2004;140:963-9.

7. Enjolras O, Riche MC, Merland JJ, Escande JP. Management of alarming hemangiomas in infancy: a review of 25 cases. Pediatrics. 1991:87:582-3.

8. Bennett $M L$, Fleischer $A B J r$, Chamlin SL, Frieden IJ. Oral corticosteroid use is effective for cutaneous hemangiomas: an evidence-based evaluation. Arch Dermatol. 2001;137:1208-13.

9. Boon LM, MacDonald DM, Mulliken JB. Complications of systemic corticosteroid therapy for problematic hemangioma. Plast Reconstr Surg. 1999;104:1616-23.

10. Tanner JL, Dechert MP, Frieden IJ. Growing up with a facial hemangioma: parent and child coping and adaptation. Pediatrics. 1998;101:446-52. 
